\title{
ON THE COMPUTATION OF DARBOUX FIRST INTEGRALS OF A CLASS OF PLANAR POLYNOMIAL VECTOR FIELDS
}

\author{
A. FERRAGUT, C. GALINDO AND F. MONSERRAT
}

\begin{abstract}
We study the class of planar polynomial vector fields admitting Darboux first integrals of the type $\prod_{i=1}^{r} f_{i}^{\alpha_{i}}$, where the $\alpha_{i}$ 's are positive real numbers and the $f_{i}$ 's are polynomials defining curves with only one place at infinity. We show that these vector fields have an extended reduction procedure and give an algorithm which, from a part of the extended reduction of the vector field, computes a Darboux first integral for generic exponents.
\end{abstract}

\section{INTRODUCTION}

Complex planar polynomial differential systems are being studied since the 19th century when Darboux [18], Poincaré [42, 43], Painlevé [40] and Autonne [5] significantly contributed to this topic. Surprisingly, nowadays, the problem of characterizing integrable differential systems as above remains open. To compute a first integral is a very interesting issue because this function provides the solution curves of the system within their domain of definition, determining the phase portrait of the system.

Darboux functions are a remarkable family of multi-valued functions. They have the following shape:

$$
H:=\prod_{i=1}^{p} f_{i}^{\lambda_{i}} \prod_{j=1}^{q} \exp \left(\frac{h_{j}}{g_{j}}\right)^{\mu_{j}},
$$

where $f_{i}$, and $g_{j}$ and $h_{j}$ are bivariate complex polynomials and $\lambda_{i}$ and $\mu_{j}$ complex numbers.

Following [20], the Darboux theory of integrability states that if a planar differential system has $p$ invariant algebraic curves whose equations are $f_{i}=0$, with cofactors $k_{i}$, $1 \leq i \leq p$, and $q$ exponential factors $\exp \left(\frac{h_{j}}{g_{j}}\right)$ with cofactors $\ell_{j}, 1 \leq j \leq q$, such that $\sum_{i=1}^{p} \lambda_{i} k_{i}+\sum_{j=1}^{q} \mu_{j} \ell_{j}=0$ for some complex numbers $\left\{\lambda_{i}\right\}_{i=1}^{p}$ and $\left\{\mu_{j}\right\}_{j=1}^{q}$, not all zero, then the function $H$ displayed in (1) is a first integral of the system.

A particular and desirable type of Darboux functions are rational functions because when $H=f / g$ is a first integral, all the invariant curves of the system are algebraic and are determined from the equations $\lambda f+\beta g=0$, where the pair $(\lambda: \beta)$ runs over the complex projective line. The so-called Poincaré problem arose when Poincaré in [43] remarked that to decide on the algebraic integrability of a differential equation of the first order and the first degree, one only needs to obtain an upper bound of the degree of the integral. Therefore this problem looks for a bound of the degree of the first integral in terms of the degree of the polynomial system, and it has generated a lot of literature.

2010 Mathematics Subject Classification. 34A34; 34C05; 34C08; 14C21.

Key words and phrases. Planar polynomial vector field, Darboux first integral, reduction of singularities, curve with only one place at infinity.

Partially supported by the Spanish Government Ministerio de Economía, Industria y Competitividad (MINECO-FEDER), grants MTM2015-65764-C3-2-P, MTM2016-81735-REDT, MTM2016-81932-REDT and MTM2016-77278-P, as well as by Universitat Jaume I grants UJI-B2018-10 and P1-1B2015-16. 
Although it is known that this upper bound does not exist in general, in some cases it can be computed. For instance when the associated vector field has only non-degenerated [43] or nodal type [15] singularities, or when its reduction uniquely admits a non-invariant exceptional divisor [27]. Notice also that, when a bound of the degree of the rational first integral is known, efficient algorithms to compute that integral have been described $[25,8]$.

Recently in [24], the authors considered a family of planar polynomial differential systems $\mathscr{F}$ formed by those systems admitting a polynomial first integral which factorizes as a product of bivariate polynomials, each of them defining a curve with only one place at infinity. These first integrals were called well-behaved at infinity, WAI for short. A plane curve has only one place at infinity when it meets the line at infinity in a unique point where it is reduced and unibranched. Abhyankar and Moh $[1,2,3]$ introduced these curves. They have a very good local-global behavior, which makes them useful when studying some algebraic and geometric problems [11, 12, 21, 22, 26]. In addition, many interesting (but hard to compute) tools, introduced for improving the knowledge of the algebraic varieties, are much easier to describe when one considers surfaces having a close relation with curves with only one place at infinity [45, 28, 39, 29].

Returning to the family $\mathscr{F}$ of differential systems, we proved in [24] that when a vector field, or equivalently a 1 -form $\omega$, corresponds to a system $S$ in $\mathscr{F}$, then a bound for the degree of the polynomial first integral can be computed from the knowledge of a part of the Seidenberg reduction of $\omega$. Furthermore, from this reduction, we are able to decide whether $S$ belongs to $\mathscr{F}$ or not and, in the positive case, to obtain the corresponding first integral. This solves the problem of deciding if a system has a polynomial first integral given by (natural) powers of curves with only one place at infinity.

We want to study whether a similar procedure can be performed for families of polynomial differential systems having a non-polynomial Darboux first integral defined by curves with only one place at infinity. So, in this paper, we consider a new family $\mathscr{D}$ of planar polynomial differential systems having what we call a Darboux positive well-behaved at infinity (DPWAI) first integral. This family satisfies $\mathscr{D} \cap \mathscr{F}=\emptyset$ and roughly speaking (see Definition 4.1 for the precise concept) a DPWAI function is a (multi-valued) function $H=\prod_{i=1}^{r} f_{i}^{\alpha_{i}}$, where $f_{i}, 1 \leq i \leq r$, are bivariate polynomials defining plane curves $C_{i}$ with only one place at infinity and satisfying that each one of them does not belong to the pencil at infinity defined by any other. In addition, the values $\alpha_{i}, 1 \leq i \leq r$, are positive real numbers satisfying a certain condition which holds when they are linearly independent over the rational numbers.

The Darboux theorem shows that if we have enough invariant algebraic curves, then a (Darboux) first integral is guaranteed (see [18] and an improvement in [16]). In fact the problem of finding Darboux first integrals is essentially the problem of finding invariant algebraic curves. Once these invariant algebraic curves are computed, and hence their cofactors are known, the existence of a Darboux first integral depends on whether or not there exists a linear combination of these cofactors which vanishes. This is, of course, a trivial problem.

But the problem of finding these invariant algebraic curves is not solved. There are some rather efficient algorithms in this direction besides the direct resolution (see for example [25]). The algorithm that we provide here uses the singularities at infinity to find these invariant algebraic curves, and therefore combines local behavior (singular points) with global behavior (invariant curves).

The main result in this paper is an algorithm whose input is a planar differential system $\mathbf{X}$ and whose output is either a first integral of $\mathbf{X}$ or "0". The output is a DPWAI first 
integral when $\mathbf{X}$ belongs to $\mathscr{D}$ and its first integral has generic exponents (see Definition 4.4 ); if the output is " 0 " then either $\mathbf{X}$ does not belong to $\mathscr{D}$, or it belongs to $\mathscr{D}$ but the exponents of the first integral are not generic. Vector fields admit a (possibly infinite) extended reduction of singularities (see Definition 4.3). This extended reduction shows that simple singularities whose quotient of eigenvalues is a positive irrational number can be "simplified" by an infinite sequence of point blowing-ups and represented by means of a proximity graph (see Subsection 2.4 and Section 4). Our algorithm uses a (finite) part of that extended reduction and, also, when $\mathbf{X}$ belongs to $\mathscr{D}$ and its first integral has generic exponents, determines the complete extended reduction over the line at infinity. Notice that, with this algorithm, we are able to compute much more Darboux first integrals (which are not rational) than in [24].

The algorithm has two steps. The first one, Algorithm 5.3, uses the mentioned part of the extended reduction to get candidates to polynomials defining the invariant curves $C_{i}$, and the second one computes the exponents $\alpha_{i}$ by using Darboux theory of integrability (Theorem 5.1). We think that, in practice, our algorithm works for any system in $\mathscr{D}$; however, due to our algebraic techniques, we can only guarantee that it computes a Darboux first integral when the exponents $\left\{\alpha_{i}\right\}_{i=1}^{r}$ are generic.

Section 5 provides the mentioned algorithm together with an example showing how it works. The ingredients we need to develop the paper are given in Section 2. WAI first integrals are recalled in Section 3 and Section 4 introduces the concept of extended reduction of a vector field and describes it for vector fields in $\mathscr{D}$ (generic exponents).

\section{Preliminaires}

Let $\mathbf{X}$ be a planar complex polynomial differential system defined by

$$
\dot{x}=p(x, y), \quad \dot{y}=q(x, y),
$$

where $p$ and $q$ are polynomials with complex coefficients in the indeterminates $x, y$ and $\operatorname{gcd}(p, q)=1$. In the sequel $\mathbf{X}$ also denotes the corresponding vector field $\mathbf{X}=p \frac{\partial}{\partial x}+q \frac{\partial}{\partial y}$.

Recall that a function $H=H(x, y)$ (may be multi-valued) is a first integral of $\mathbf{X}$ if $H$ is constant along any solution of the system. If $H \in \mathcal{C}^{1}$, then the equality

$$
\mathbf{X} H=p \frac{\partial H}{\partial x}+q \frac{\partial H}{\partial y}=0
$$

holds whenever $H$ is defined.

In this paper, we will study the family of vector fields $\mathbf{X}$ admitting a particular class of Darboux first integrals. We devote this section to summarize some concepts and properties we will need.

We have introduced the polynomial differential system (2) by using affine coordinates; however, in this paper, we will need to consider its complex projectivization. Therefore we start by studing the complex projectivization $\mathcal{X}$ of the vector fields $\mathbf{X}$ and their corresponding 1-forms.

2.1. Polynomial vector fields on $\mathbb{C P}^{2}$. Let us consider the complex projective plane $\mathbb{C P}^{2}$, with homogeneous coordinates $X, Y, Z$, and homogeneous polynomials of degree $d+1$ without common factors $A_{1}, A_{2}$, and $A_{3}$ in $\mathbb{C}[X, Y, Z]$. The 1-form

$$
\Omega=A_{1} d X+A_{2} d Y+A_{3} d Z
$$

(of degree $d+1$ ) is said to be projective if it satisfies the so-called Euler condition:

$$
X A_{1}+Y A_{2}+Z A_{3}=0 .
$$


This projective 1-form determines (and is determined by), up to addition of a multiple of the radial vector field $X \frac{\partial}{\partial X}+Y \frac{\partial}{\partial Y}+Z \frac{\partial}{\partial Z}$, a homogeneous polynomial vector field $\mathcal{X}=P_{1} \frac{\partial}{\partial X}+P_{2} \frac{\partial}{\partial Y}+P_{3} \frac{\partial}{\partial Z}$, where $P_{1}, P_{2}$ and $P_{3}$ are homogeneous polynomials in $\mathbb{C}[X, Y, Z]$ of the same degree $d$ and without common factors (see, for instance, Section 1 of [13]). $\mathcal{X}$ gives a field of directions on $\mathbb{C P}^{2}$ and, by abuse of language, is named vector field on $\mathbb{C P}^{2}$ [41, page 1387]. The number $d$ is the degree of $\mathcal{X}$.

Let $F$ be a homogeneous polynomial with complex coefficients and indeterminates $X, Y, Z$. The curve on $\mathbb{C P}^{2}$ defined by the equation $F=0$ is invariant by the vector field $\mathcal{X}$ if there exists a homogeneous polynomial $K \in \mathbb{C}[X, Y, Z]$ of degree $d-1$ such that

$$
\mathcal{X} F=P_{1} \frac{\partial F}{\partial X}+P_{2} \frac{\partial F}{\partial Y}+P_{3} \frac{\partial F}{\partial Z}=K F .
$$

The polynomial $K$ is called the cofactor of $F$.

The singular points of $\mathcal{X}$ are those in the projective plane that satisfy the following system of equations:

$$
A_{1}(X, Y, Z)=0, \quad A_{2}(X, Y, Z)=0, \quad A_{3}(X, Y, Z)=0 .
$$

Considering affine coordinates $x=X / Z$ and $y=Y / Z$ in the affine chart defined by $Z \neq 0$, the homogeneous polynomial vector field $\mathcal{X}$ restricts to the affine vector field

$$
\mathbf{X}=a_{2}(x, y) \frac{\partial}{\partial x}-a_{1}(x, y) \frac{\partial}{\partial y},
$$

where $a_{1}(x, y)=A_{1}(x, y, 1)$ and $a_{2}(x, y)=A_{2}(x, y, 1) . \mathbf{X}$ is also defined by the 1 -form $\omega=a_{1}(x, y) d x+a_{2}(x, y) d y$. Taking into account Equality (3), one can recover $\Omega$ from the affine 1 -form $\omega$.

To provide the system (2) is equivalent to give the 1 -form

$$
p(x, y) d y-q(x, y) d x
$$

and, therefore, a homogeneous polynomial vector field $\mathcal{X}$ which is called the complex projectivization of $\mathbf{X}$.

Recall that an invariant algebraic curve of the vector field $\mathbf{X}$ is an affine algebraic curve with local equation $f(x, y)=0, f \in \mathbb{C}[x, y]$, such that $\mathbf{X} f=k f$, where $k \in \mathbb{C}[x, y]$ is called the cofactor of $f=0$. Now, if $f(x, y)$ has degree $n \in \mathbb{N}$, then $F(X, Y, Z)=$ $Z^{n} f(X / Z, Y / Z)=0$ is an invariant algebraic curve of the vector field $\mathcal{X}$ with cofactor $K(X, Y, Z)=Z^{d-1} k(X / Z, Y / Z)$.

Next, we give a short overview of the blow-up technique to reduce the singularities of a planar vector field, which will be a key element in this paper.

2.2. Reduction of singularities. The singularities of planar vector fields can be reduced by blowing-up (see [24, Section 4.1] for details). This procedure, due to Seidenberg, performs algebraic modifications and gives rise to a simpler vector field on a different to $\mathbb{C P}^{2}$ surface, which makes easier the study of the original vector field $[44,19,4]$. Next we recall the concepts of singularity and simple singularity.

Definition 2.1. A point $O \in \mathbb{C}^{2}$ is called a singularity of a polynomial vector field $\mathbf{X}=p(x, y) \frac{\partial}{\partial x}+q(x, y) \frac{\partial}{\partial y},\{x, y\}$ being local coordinates at $O$, if the multiplicity of $\mathbf{X}$ at $O$ (that is, the minimum of the orders of $p=p(x, y)$ and $q=q(x, y)$ at $O$ ) is strictly positive. The singularity $O$ is simple in case $\mathbf{X}$ has multiplicity 1 at $O$ and the matrix 
defined by the first nonzero jet $p_{1} d y-q_{1} d x$ of the differential 1-form $p d y-q d x$,

$$
\left(\begin{array}{ll}
\frac{\partial p_{1}}{\partial x} & \frac{\partial p_{1}}{\partial y} \\
\frac{\partial q_{1}}{\partial x} & \frac{\partial q_{1}}{\partial y}
\end{array}\right),
$$

has eigenvalues $\lambda_{1}, \lambda_{2}$ satisfying either $\lambda_{1} \lambda_{2} \neq 0$ and $\frac{\lambda_{1}}{\lambda_{2}} \notin \mathbb{Q}^{+}$, or $\lambda_{1} \lambda_{2}=0$ and $\lambda_{1}^{2}+\lambda_{2}^{2} \neq 0$. A non-simple singularity is named ordinary.

Seidenberg's reduction theorem [44] (see also [9]) shows that the singularities of a planar vector field can be transformed into simple singularities by means of blowing-ups:

Theorem 2.2. Let $O \in \mathbb{C}^{2}$ be an isolated singularity of a polynomial vector field $\mathbf{X}=$ $p \frac{\partial}{\partial x}+q \frac{\partial}{\partial y}$ in $\mathbb{C}^{2}$. Then there exists a finite sequence of blowing-ups of closed points of the successively obtained surfaces which starts blowing-up $O, \pi: \mathcal{Z} \rightarrow \mathbb{C P}^{2}$, such that the singularities of the strict transform of the vector field $\mathbf{X}$ at the surface $\mathcal{Z}$ are simple.

From a global point of view, Seidenberg's result states that, given an homogeneous polynomial vector field $\mathcal{X}$ on $\mathbb{C P}^{2}$, there exists a set of points (or configuration, according with a forthcoming definition)

$$
\mathcal{S}(\mathcal{X})=\left\{Q_{0}, Q_{1}, \ldots, Q_{n}\right\}
$$

such that $Q_{0} \in X_{0}:=\mathbb{C P}^{2}, \pi_{Q_{i-1}}: \mathrm{Bl}_{Q_{i-1}}\left(X_{i-1}\right) \rightarrow X_{i-1}$ is the blowing-up of $X_{i-1}$ centered at $Q_{i-1}, Q_{i} \in \mathrm{Bl}_{Q_{i-1}}\left(X_{i-1}\right)=: X_{i}$ for $1 \leq i \leq n$, and the composition

$$
\pi=\pi_{Q_{0}} \circ \cdots \circ \pi_{Q_{n}}: \mathcal{Z}:=X_{n+1} \longrightarrow \mathbb{C P}^{2}
$$

is such that every singularity of the strict transform $\tilde{\mathcal{X}}$ of $\mathcal{X}$ in $\mathcal{Z}$ is simple. We call $\mathcal{S}(\mathcal{X})$ the singular configuration of $\mathcal{X}$. For an explicit local description of the reduction of singularities and the obtention of the singular configuration, including a detailed example, see Section 4 of [24].

A singularity of a vector field is dicritical if infinitely many solutions pass through it (see [24, Definition 1] for an equivalent definition that allows us to detect the dicritical character of a singularity from the reduction process). In this paper, we will denote by $\mathcal{D}(\mathcal{X})$ the dicritical configuration of $\mathcal{X}$, that is the set of dicritical singularities in $\mathcal{S}(\mathcal{X})$ of the strict transforms along $\pi$ of the vector field $\mathcal{X}$ (see [24, page 360] for the definition of strict transform of a vector field and the straightforward translation of the concept of dicritical singularity to these vector fields over surfaces obtained by blowing-up).

In the following two subsections we recall the concepts of configuration of infinitely near points and its proximity graph, and that of proximity graph defined by a positive real number.

2.3. Proximity graph of a configuration: singular and dicritical graphs. Consider a point $P$ of a smooth complex projective surface. Blowing-up $P$, one obtains an exceptional divisor $E_{P}$ usually called the first infinitesimal neighborhood of $P$ and, for each $i>0$, the $i$ th infinitesimal neighborhood of $P$ is the first infinitesimal neighborhood of some point belonging to the $(i-1)$ th infinitesimal neighborhood of $P$. A point $R \neq P$ in some infinitesimal neighborhood of $P$ is proximate to $P$ whenever it is in the strict transform of the exceptional divisor $E_{P}$. Also if $R$ is in the intersection of the strict transforms of two exceptional divisors (that is, if it is proximate to two points), then we say that $R$ is satellite. Otherwise $R$ is free. 
The set of points that are infinitely near to $P$ is given by those points belonging to the $i$ th infinitesimal neighborhood of $P$, for some $i>0$. This set admits a natural ordering: a point $Q$ precedes another point $S$ if $S$ is infinitely near to $Q$. Also, for us, a point is infinitely near to itself.

A configuration of infinitely near points (or, simply, a configuration) of a complex surface $X_{0}$ (it could be $\mathbb{C P}^{2}$ ) is a (finite or infinite) set of points

$$
\mathcal{C}=\left\{P_{0}, P_{1}, \ldots\right\}
$$

such that $P_{0} \in X_{0}$ and, for all $i \geq 1, P_{i}$ belongs to the blow-up $X_{i}$ of $X_{i-1}$ with center at $P_{i-1}$.

The proximity graph of $\mathcal{C}, \Gamma_{\mathcal{C}}$, is a directed graph with labeled edges whose set of vertices is $\mathcal{C}$ and whose edges are given by the pairs $(P, Q)$ such that $Q$ is proximate to $P$. The edges $(P, Q)$ have two different labels according to $Q$ belongs, or not, to the first infinitesimal neighborhood of $P$. When drawing $\Gamma_{\mathcal{C}}$, the labels of the first type are represented by a straight segment and those of the second type by a curved-dotted segment (joining $P$ and $Q$ in both cases). For convenience, we delete arrows (our segments are always ascendent) and those curved-dotted edges that can be deduced from others; notice that if $P, R, Q$ are points in $\mathcal{C}$ such that $P$ precedes $R, R$ precedes $Q$ and $Q$ is proximate to $P$, then $R$ is also proximate to $P$.

If $\mathcal{X}$ is, as above, a projective vector field on $\mathbb{C P}^{2}$, the proximity graph $\Gamma_{\mathcal{S}(\mathcal{X})}$ (respectively, $\Gamma_{\mathcal{D}(\mathcal{X})}$ ) is called the singular graph (respectively, dicritical graph) of $\mathcal{X}$.

2.4. The proximity graph defined by a positive real number. Let $X_{0}$ be a complex surface, $P$ a point in $X_{0}$ and $C$ a germ of curve on the local ring of $X_{0}$ at $P$ having only one analytic branch. Assuming that $P$ is singular, one can determine the configuration of infinitely near points $\mathcal{D}_{C}=\left\{P_{0}, P_{1}, \ldots, P_{s}\right\}$ such that:

(i) $P_{0}=P$ and, for all $i \geq 1, P_{i}$ is the point where the exceptional divisor $E_{P_{i-1}}$ meets the strict transform of $C$.

(ii) The composition of the blowing-ups centered at the points of $\mathcal{D}_{C}$ gives rise to a minimal embedded resolution of the singularity of $C$ at $P$.

For $i \geq 0$, let $m_{i}$ denote the multiplicity at $P_{i}$ of the strict transform of $C$. Each one of these numbers $m_{i}$ satisfies the so-called proximity equalities: $m_{i}=1$ if $i=s$ and, otherwise, $m_{i}=\sum m_{j}$, where the sum runs over those indices $j$ such that $P_{j}$ is proximate to $P_{i}$ [14]. Notice that the $(s+1)$-tuple of multiplicities $\left(m_{0}, m_{1}, \ldots, m_{s}\right)$ uniquely determines the proximity graph of the configuration $\mathcal{D}_{C}$.

If the singularity of $C$ at $P$ has only one Puiseux pair (i.e., the minimal embedded resolution is obtained by blowing-up some free points and, afterwards, finitely many satellite points), then the sequence of multiplicities of $C$ at $P$ has the shape

$$
\left(r_{0\left(c_{0}\right)}, r_{1\left(c_{1}\right)}, \ldots, r_{\ell\left(c_{\ell}\right)}=1_{\left(c_{\ell}\right)}\right),
$$

where the subindices $\left(c_{i}\right)_{i=0}^{\ell}$ indicate the number of times that each multiplicity is repeated. Moreover, the numbers $c_{i}$ come from the continued fraction expansion of the rational number

$$
\frac{\sum_{i=0}^{s} m_{i}^{2}}{m_{0}^{2}}=\left[c_{0} ; c_{1}, \ldots, c_{\ell}\right]:=c_{0}+\frac{1}{c_{1}+\frac{1}{c_{2}+\ldots \frac{1}{c_{\ell}}}} ;
$$

$r_{0}=m_{0}$ and $r_{1}, r_{2}, \ldots, r_{\ell}$ are the successive remainders appearing when the Euclidean algorithm is applied to $\sum_{i=0}^{s} m_{i}^{2}$ and $m_{0}^{2}$ [14]. Hence, in this case, the proximity graph of $\mathcal{D}_{C}$ is determined by the rational number $\frac{\sum_{i=0}^{s} m_{i}^{2}}{m_{0}^{2}}$. Similarly, the continued fraction 
expansion of any positive rational number $\beta$ determines a proximity graph, which we will denote by $\operatorname{Prox}(\beta)$.

Now, let $\gamma$ be a positive irrational number and consider its (infinite) continued fraction expansion

$$
\gamma=\left[c_{0} ; c_{1}, c_{2}, \ldots\right]:=c_{0}+\frac{1}{c_{1}+\frac{1}{c_{2}+\ldots}} .
$$

For each $i \geq 0$ set $\beta_{i}:=\left[c_{0} ; c_{1}, \ldots, c_{i}\right]$. Each proximity graph $\operatorname{Prox}\left(\beta_{i+1}\right)$ can be obtained from $\operatorname{Prox}\left(\beta_{i}\right)$ by adding new vertices and labeled edges; then $\operatorname{Prox}\left(\beta_{i}\right)$ can be regarded as a subgraph of $\operatorname{Prox}\left(\beta_{i+1}\right)$. We define the proximity graph given by $\gamma, \operatorname{Prox}(\gamma)$, as the (infinite) directed graph with labeled edges whose set of vertices (respectively, edges) is the union of the sets of vertices (respectively, edges) of the graphs $\operatorname{Prox}\left(\beta_{i}\right), i \geq 0$, keeping the labels of the edges.

Remark 2.3. Consider the category $\mathfrak{C}$ whose objects are the (finite and infinite) directed graphs with labeled edges (two labels) and whose morphisms are the label-preserving morphisms of graphs. Let $\gamma$ and $\beta_{i}, i \geq 0$, be as before. For each pair of indexes $i, j \geq 0$ such that $i \leq j$ there exists an obvious (inclusion) morphism of labeled graphs $f_{i j}: \operatorname{Prox}\left(\beta_{i}\right) \rightarrow \operatorname{Prox}\left(\beta_{j}\right)$; these morphisms allow us to give a direct system [35, III, §10]

$$
\left\{\left(\operatorname{Prox}\left(\beta_{i}\right)\right)_{i \geq 0} ;\left(f_{i j}\right)_{0 \leq i \leq j}\right\}
$$

in the category $\mathfrak{C}$. From our construction, it is straightforward to check that $\operatorname{Prox}(\gamma)$ is the direct limit of the above mentioned direct system.

Next, in Figure 1, we show the shape of the proximity graph $\operatorname{Prox}(\gamma)$.

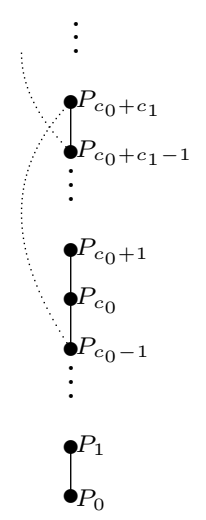

Figure 1. Proximity graph defined by $\gamma$.

The relation between elimination of base points of certain linear systems and the reduction of singularities of planar vector fields having a rational first integral supports some reasonings in this paper. So we conclude this section with a brief of those concepts close to linear systems we will use.

2.5. Linear systems and pencils. For any natural number $m>0$, let us denote by $\mathbb{C}_{m}[X, Y, Z]$ the (projective) space of homogeneous polynomials of degree $m$ in the indeterminates $X, Y, Z$. A linear system on $\mathbb{C P}^{2}$ will be the set of algebraic curves defined by a linear space of polynomials in $\mathbb{C}_{m}[X, Y, Z]$ for some natural number $m>0$. Notice that 
a linear system has structure of projective space. A pencil is a linear system of projective dimension 1.

Next, we recall the definition of cluster of $\mathbb{C P}^{2}$ and other related concepts [14] (see also [10] and [24]).

Definition 2.4. A (weighted) cluster of infinitely near points (or, simply, a cluster) of $\mathbb{C P}^{2}$ is a pair $(\mathcal{C}, \mathbf{m})$ where $\mathcal{C}=\left\{Q_{0}, Q_{1}, \ldots, Q_{h}\right\}$ is a configuration of infinitely near points of $\mathbb{C P}^{2}$ and $\mathbf{m}=\left(m_{0}, m_{1}, \ldots, m_{h}\right) \in \mathbb{N}^{n}, \mathbb{N}$ being the set of positive integers.

We desire to consider linear systems determined by clusters. To this purpose, we require some notations and concepts. For the convenience of the reader, we start by recalling the concept of virtual transform (respectively, passing virtually) of a curve at (respectively, through) a point of a cluster.

Fix a cluster $\mathcal{K}=(\mathcal{C}, \mathbf{m})$, for each $Q_{i} \in \mathcal{C}$, we set

$$
\ell\left(Q_{i}\right):=\operatorname{card}\left\{Q_{j} \in \mathcal{C} \mid Q_{i} \text { is infinitely near to } Q_{j}\right\} .
$$

Consider a point $Q_{k} \in \mathcal{C}$ and an algebraic curve $C$ on $\mathbb{C P}^{2}$.

Assume first that $\ell\left(Q_{k}\right)=1$. Pick local coordinates $(x, y)$ at $Q_{k}$ and a local equation of $C, f(x, y)=0$. The virtual transform of $C$ at $Q_{k}$ with respect to the cluster $\mathcal{K}, C_{Q_{k}}^{\mathcal{K}}$, is the (local) curve defined by $f(x, y)=0$. The degree of the first non-zero jet of $f(x, y)$ is named the multiplicity of $C_{Q_{k}}^{\mathcal{K}}$ at $Q_{k}$ and denoted by $m_{Q_{k}}\left(C_{Q_{k}}^{\mathcal{K}}\right)$. Finally the curve $C$ passes virtually through $Q_{k}$ with respect to $\mathcal{K}$ whenever $m_{Q_{k}}\left(C_{Q_{k}}^{\mathcal{K}}\right) \geq m_{k}$.

Consider now the case where $\ell\left(Q_{k}\right)>1$. Set $Q_{j} \in \mathcal{C}$ such that $Q_{k}$ is in the first infinitesimal neighborhood of $Q_{j}$ and suppose (by induction) that $C$ passes virtually through $Q_{j}$ with respect to $\mathcal{K}$. Pick local coordinates $(x, y)$ at $Q_{j}$ and $f(x, y)=0$ a local equation of $C_{Q_{j}}^{\mathcal{K}}$. The point $Q_{k}$ belongs to the surface obtained by blowing-up $Q_{j}$ and thus $Q_{k}=(0, \delta)$ (respectively, $\left.Q_{k}=(\delta, 0)\right), \delta \in \mathbb{C}$, in local coordinates $(x, t=y / x)$ (respectively, $(s=x / y, y))$. Then, the virtual transform of $C$ at $Q_{k}$ with respect to $\mathcal{K}, C_{Q_{k}}^{\mathcal{K}}$, is the curve defined by $x^{-m_{j}} f(x, x(t+\delta))=0$ when considering the first coordinates and that defined by $y^{-m_{j}} f((s+\delta) y, y)=0$ otherwise. As above, the multiplicity of $C_{Q_{k}}^{\mathcal{K}}$ at $Q_{k}$ is denoted by $m_{Q_{k}}\left(C_{Q_{k}}^{\mathcal{K}}\right)$ and $C$ passes virtually through $Q_{k}$ with respect to $\mathcal{K}$ if $m_{Q_{k}}\left(C_{Q_{k}}^{\mathcal{K}}\right) \geq m_{k}$. When the curve $C$ passes virtually through $Q_{i}$ with respect to $\mathcal{K}$ for all $Q_{i} \in \mathcal{K}$, we say that $C$ passes virtually through $\mathcal{K}$.

Now we define the above mentioned linear systems. Consider a cluster $\mathcal{K}=(\mathcal{C}, \mathbf{m})$ of $\mathbb{C P}^{2}$ and a positive integer $m$.

Definition 2.5. The linear system defined by the pair $(m, \mathcal{K}), \mathcal{L}_{m}(\mathcal{K})$, is the linear system of curves on $\mathbb{C P}^{2}$ passing virtually through $\mathcal{K}$ and given by homogeneous polynomials of degree $m$.

The following concept will be useful in this paper.

Definition 2.6. Let $Z$ be the complex surface defined by a (finite) configuration of infinitely near points of $\mathbb{C P}^{2}, \mathcal{C}, \pi: Z \rightarrow \mathbb{C P}^{2}$ the corresponding blowing-up map and $C$ an algebraic curve on $\mathbb{C P}^{2}$. The strict transform $\tilde{C}$ of $C$ on $Z$ is the image of $C$ by the birational map $\pi^{-1}$.

Let $n$ be a positive integer and $F_{1}, F_{2}, \ldots, F_{s}$ linearly independent polynomials in $\mathbb{C}_{n}[X, Y, Z]$ with no common factor. Set $\mathcal{L}=\mathbb{P} V$ the linear system on $\mathbb{C P}^{2}$ associated to the linear space over $\mathbb{C}, V=\left\langle F_{1}, F_{2}, \ldots, F_{s}\right\rangle$, generated by the polynomials $F_{i}, 1 \leq i \leq s$. 
Then there exist linear subspaces $\mathcal{H}_{i} \subsetneq \mathbb{C P}^{s-1}$ of $\mathbb{C P}^{s-1}, 1 \leq i \leq t$, and a configuration of infinitely near points of $\mathbb{C P}^{2}$, which we denote by $\mathcal{B P}(\mathcal{L})$, such that:

a) The multiplicities at each $R \in \mathcal{B P}(\mathcal{L})$ of the strict transforms of the curves $\alpha_{1} F_{1}+$ $\alpha_{2} F_{2}+\cdots+\alpha_{s} F_{s}=0$, where $\bar{\alpha}=\left(\alpha_{1}, \alpha_{2}, \ldots, \alpha_{s}\right)$ runs through $\mathcal{A}:=\mathbb{C P}^{s-1} \backslash \bigcup_{i=1}^{t} \mathcal{H}_{i}$, are the same, denoted by $\operatorname{mult}_{R}(\mathcal{L})$.

b) The virtual transforms of the curves $\sum \alpha_{i} F_{i}=0, \bar{\alpha} \in \mathcal{A}$, with respect to the cluster

$$
\left(\mathcal{B P}(\mathcal{L}),\left(\operatorname{mult}_{R}(\mathcal{L})\right)_{R \in \mathcal{B} \mathcal{P}(\mathcal{L})}\right)
$$

have no common point; these curves are called generic curves of $\mathcal{L}$.

Observe also that the set $\bigcup_{i=1}^{t} \mathcal{H}_{i}$ is finite in case $\mathcal{L}$ is a pencil.

Definition 2.7. With the above notations, the pair $(\mathcal{B P}(\mathcal{L}), \mathbf{m})$, where

$$
\mathbf{m}=\left(\operatorname{mult}_{Q}(\mathcal{L})\right)_{Q \in \mathcal{B} \mathcal{P}(\mathcal{L})},
$$

is a cluster of infinitely near points of $\mathbb{C P}^{2}$ called the cluster of base points of $\mathcal{L}$.

\section{REduction of Singularities of A Vector Field With WAI FiRst Integral}

Plane curves with only one place at infinity were initially considered in $[1,2,3]$. We begin by stating the definition.

Definition 3.1. An algebraic projective curve $C$ defined by a homogeneous polynomial $F \in \mathbb{C}[X, Y, Z]$ has only one place at infinity if $C$ meets the line at infinity $Z=0$ at only one point, where it is reduced and unibranched.

Next we introduce the concept of well-behaved at infinity (WAI) first integral.

Definition 3.2. A complex planar polynomial differential system (or a vector field) $\mathbf{X}$ has a WAI first integral whenever $\mathbf{X}$ admits a polynomial

$$
H=\prod_{i=1}^{r} f_{i}^{n_{i}},
$$

as a first integral, $r$ and $n_{i}, 1 \leq i \leq r$, being positive integers and $f_{i}, 1 \leq i \leq r$, complex bivariate polynomials of degree $d_{i} \in \mathbb{N}$ such that each complex projective curve $C_{i}$ on $\mathbb{C P}^{2}$ defined by the polynomial $F_{i}(X, Y, Z)=Z^{d_{i}} f_{i}(X / Z, Y / Z)$ has only one place at infinity.

Along this paper we will also assume that, if $H$ is WAI first integral, then

$$
\operatorname{gcd}\left(n_{1}, n_{2}, \ldots, n_{r}\right)=1, \quad r \geq 2,
$$

and the following condition is fulfilled:

$$
f_{i}-\lambda f_{j} \notin \mathbb{C} \text { for all } i, j \in\{1,2, \ldots, r\} \text { such that } i \neq j \text { and for all } \lambda \in \mathbb{C} \text {. }
$$

For $1 \leq j \neq i \leq r$, write

$$
\rho_{j i}:=\sum_{Q}\left(C_{j}, C_{i}\right)_{Q}
$$

where $Q$ runs through the common points of $C_{j}$ and $C_{i}$ outside the line at infinity (defined by $Z=0)$, and $\left(C_{j}, C_{i}\right)_{Q}$ is the intersection multiplicity between $C_{i}$ and $C_{j}$ at $Q$.

We will assume in the remaining of this section that $\mathbf{X}$ is a complex planar polynomial vector field (whose complex projectivization to $\mathbb{C P}^{2}$ is $\mathcal{X}$ ) which admits a WAI first integral. By [24, Corollary 1], the dicritical configuration $\mathcal{D}(\mathcal{X})$ coincides with the configuration of the cluster of base points of the pencil $\mathbb{P} V$, where $V=\left\langle\prod_{i=1}^{r} F_{i}^{n_{i}}, Z^{d}\right\rangle$ and $d=\sum_{i=1}^{r} n_{i} d_{i}, d_{i}:=\operatorname{deg}\left(F_{i}\right)$. Taking advantage of the study of this cluster performed in 
[12], we state the following result, which provides a very specific information about the dicritical configuration $\mathcal{D}(\mathcal{X})$.

Proposition 3.3. Let $\mathbf{X}$ and $\mathcal{X}$ be as above. Then

$$
\mathcal{D}(\mathcal{X})=\bigcup_{i=1}^{r}\left(\mathcal{B P}\left(\mathcal{P}_{i}\right) \cup\left\{S_{i}\right\} \cup \mathcal{L}_{i}\right)
$$

where, for each $i=1,2, \ldots, r$ :

(a) $\mathcal{B P}\left(\mathcal{P}_{i}\right)$ is the configuration of base points of the pencil $\mathcal{P}_{i}$ defined by the non-zero linear combinations of the polynomials $F_{i}$ and $Z^{d_{i}}$.

(b) The configuration $\mathcal{B P}\left(\mathcal{P}_{i}\right)$, ordered by the relation "to be infinitely near to", has only one maximal point $Q_{i}$. Moreover, the strict transform of the curve $C_{i}$ with equation $F_{i}=0$ meets the exceptional divisor $E_{Q_{i}}$ at a unique point $S_{i}$. The local 1 -form at $S_{i}$ defining the strict transform of $\mathcal{X}$ has the shape $\delta_{i} u d t-n_{i} t d u$, where $u, t$ are coordinates whose vanishing give local equations of the strict transform of $C_{i}$ at $S_{i}$ and of the exceptional divisor $E_{Q_{i}}$, and where

$$
\delta_{i}=\sum_{\substack{j=1 \\ j \neq i}}^{r} n_{j} \rho_{j i} .
$$

Furthermore, $\left\{S_{i}\right\} \cup \mathcal{L}_{i}$ is the configuration of base points of the (local) pencil $\left(\right.$ at $\left.S_{i}\right)$ defined by the non-zero linear combinations of $u^{n_{i}}$ and $t^{\delta_{i}}$.

(c) No value $\rho_{j i}$ equals zero.

(d) The point $S_{i}$ is free.

(e) If $i \neq j$ then $S_{i}$ is not infinitely near to $S_{j}$ and $S_{j}$ is not infinitely near to $S_{i}$.

Proof. Items (a), (b), and (e) follow from [12, Lemma 1] and its proof. The sequence of blowing-ups centered at the points in $\mathcal{B P}\left(\mathcal{P}_{i}\right)$ provides an embedded resolution of the singularity of $C_{i}$ at infinity (see $[2,37]$ ) and, as a consequence, the point $S_{i}$ is free. This proves Item (d).

It remains to prove (c). Reasoning by contradiction, assume that $\rho_{j i}=0$ for some indexes $i, j \in\{1,2, \ldots, r\}$ such that $i \neq j$. Blowing-up the points in $\mathcal{B P}\left(\mathcal{P}_{i}\right)$ we get a surface $Y$. Let $\tilde{C}_{i}$ and $\tilde{C}_{j}$ be the respective strict transforms of $C_{i}$ and $C_{j}$ on $Y$. By (b) and (e) and our assumption, $\tilde{C}_{i}$ and $\tilde{C}_{j}$ do not meet, and then $\tilde{C}_{i} \cdot \tilde{C}_{j}=0$. This means, by the projection formula, that $\tilde{C}_{j}$ is contracted by the morphism defined by a basis of global sections of the sheaf $\mathcal{O}_{Y}\left(\tilde{C}_{i}\right)$ [31]. Thus $C_{j}$ must be a curve of the pencil $\mathcal{P}_{i}$, and this contradicts Condition (4), which is assumed for the WAI first integral $H$.

Remark 3.4. The configuration $\bigcup_{i=1}^{r}\left(\mathcal{B} \mathcal{P}\left(\mathcal{P}_{i}\right) \cup\left\{S_{i}\right\}\right)$ is independent of the exponents $n_{1}, n_{2}, \ldots, n_{r}$ appearing in the WAI first integral.

\section{Extended REDUCtion of Singularities AND DPWAI First integrals}

An algorithm for deciding whether a system as (2) has a WAI first integral, and computing that integral in the affirmative case, was given in [24]. This algorithm uses a part of the reduction procedure for the corresponding vector field $\mathcal{X}$. We want to find out whether there is some close procedure to compute other types of Darboux first integrals involving curves with only one place at infinity. Next we introduce a set of Darboux functions and an extended reduction of singularities of vector fields suitable for our purposes. 
Definition 4.1. A Darboux positive well-behaved at infinity (DPWAI, for short) function is a multi-valued function of the form

$$
H=\prod_{i=1}^{r} f_{i}^{\alpha_{i}}, \quad r \geq 2,
$$

where:

(1) The $f_{i}$ 's, $i \leq i \leq r$, are bivariate complex polynomials of degree $d_{i} \in \mathbb{N}$ such that the projective curve $C_{i}$, defined by $F_{i}(X, Y, Z)=Z^{d_{i}} f_{i}(X / Z, Y / Z)$, has only one place at infinity.

(2) The values $\alpha_{i}, i \leq i \leq r$, are strictly positive real numbers.

(3) The polynomials $f_{i}, 1 \leq i \leq r$, satisfy Condition (4).

(4) For all $i \in\{1,2, \ldots, n\}$, there is no positive rational number $\beta$ such that

$$
\beta \alpha_{i}=\sum_{\substack{j=1 \\ j \neq i}}^{r} \rho_{j i} \alpha_{j},
$$

where $\rho_{j i}$ is the value defined in (5).

Remark 4.2. Note that our last condition holds, in particular, when $\left\{\alpha_{i}\right\}_{i=1}^{r}$ is a linearly independent set over the field $\mathbb{Q}$.

Now we define the concept of extended reduction of singularities.

Definition 4.3. An extended reduction of singularities of an arbitrary singular polynomial vector field $\mathcal{X}$ on $\mathbb{C P}^{2}$ is a sequence of blowing-ups

$$
\cdots \rightarrow X_{i+1} \rightarrow X_{i} \rightarrow \cdots \rightarrow X_{1} \rightarrow X_{0}=\mathbb{C P}^{2}
$$

obtained by performing, first, a reduction of singularities of $\mathcal{X}$ and, then, by successively blowing-up every simple singularity of the transformed vector field whose quotient of eigenvalues is a positive real number.

This extended reduction can be regarded as a more natural procedure than Seidenberg's reduction because we keep blowing-up points while the quotient of eigenvalues is a positive real number $\gamma$. Although we can obtain an infinite sequence of blowing-ups, this sequence is completely determined by the continued fraction expansion of $\gamma$. Seidenberg applies only this procedure when $\gamma$ is rational giving rise to a finite sequence of point blowing-ups.

Let us denote by $\mathcal{E}(\mathcal{X})$ the configuration of infinitely near points of $\mathbb{C P}^{2}$ formed by the centers of the extended reduction of singularities of $\mathcal{X}$. Also, $\mathcal{E}_{\infty}(\mathcal{X})$ will denote the configuration of points in $\mathcal{E}(\mathcal{X})$ whose images on $\mathbb{C P}^{2}$ by the sequence (6) belong to the line at infinity.

Definition 4.4. We will say that a property $\mathbf{P}$ is satisfied for generic exponents $\left\{\alpha_{i}\right\}_{i=1}^{r}$ if there exists a finite set of non-zero polynomials $\left\{h_{j}\left(z_{1}, z_{2}, \ldots, z_{r}\right)\right\}_{j \in J} \subseteq \mathbb{C}\left[z_{1}, \ldots, z_{r}\right], J$ a set of indexes, fulfilling the following condition: $\mathbf{P}$ is satisfied for all $r$-tuples $\left(\alpha_{1}, \alpha_{2}, \ldots, \alpha_{r}\right)$ such that $h_{j}\left(\alpha_{1}, \ldots, \alpha_{r}\right) \neq 0$ for all $j \in J$.

Until the end of this section, we will suppose that $\mathbf{X}$ is a polynomial vector field (or differential system) of $\mathbb{C}^{2}$ having a DPWAI first integral

$$
H=\prod_{i=1}^{r} f_{i}^{\alpha_{i}} .
$$


Consider the complex projectivization $\mathcal{X}$ of $\mathbf{X}$ and set $\Omega_{\mathcal{X}}=A_{1} d X+A_{2} d Y+A_{3} d Z$ a homogeneous reduced 1-form defining $\mathcal{X}$. The following result explains how $\Omega_{\mathcal{X}}$ writes for generic exponents. See [36] for the affine version with a polynomial differential system having a generalized Darboux first integral.

Proposition 4.5. With the above notation it follows that, for generic exponents $\alpha_{1}, \ldots, \alpha_{r}$, the reduced 1-form $\Omega_{\mathcal{X}}$ is, up to multiplication by a non-zero constant, equal to

$$
\begin{aligned}
\left(\sum_{i=1}^{r} \alpha_{i} Z \prod_{\substack{j=1 \\
j \neq i}}^{r} F_{j} \frac{\partial F_{i}}{\partial X}\right) d X+\left(\sum_{i=1}^{r} \alpha_{i} Z \prod_{\substack{j=1 \\
j \neq i}}^{r} F_{j} \frac{\partial F_{i}}{\partial Y}\right) d Y & \\
& +\left(\sum_{i=1}^{r} \alpha_{i} Z \prod_{\substack{j=1 \\
j \neq i}}^{r} F_{j} \frac{\partial F_{i}}{\partial Z}-d \prod_{j=1}^{r} F_{j}\right) d Z,
\end{aligned}
$$

where $F_{i}$ is the projectivization of $f_{i}, 1 \leq i \leq r$, and $d=\sum_{i=1}^{r} \alpha_{i} \operatorname{deg}\left(F_{i}\right)$.

Proof. It is straightforward to check that $\prod_{i=1}^{r} f_{i}^{\alpha_{i}}$ is a first integral of the vector field obtained by the restriction of $\Omega_{\mathcal{X}}$ to the affine plane. So, it only remains to prove that $\Omega_{\mathcal{X}}$ is reduced.

Let $\mathcal{X}^{\prime}$ be the map which sends every element $\bar{\beta}=\left(\beta_{1}, \beta_{2}, \ldots, \beta_{r}\right) \in \mathbb{R}^{r}$ to the vector field $\mathcal{X}^{\prime}(\bar{\beta})$ on $\mathbb{C P}^{2}$ defined by the homogeneous 1 -form

$$
\begin{aligned}
\Omega\left(\beta_{1}, \beta_{2}, \ldots, \beta_{r}\right):= & \left(\sum_{i=1}^{r} \beta_{i} Z \prod_{\substack{j=1 \\
j \neq i}}^{r} F_{j} \frac{\partial F_{i}}{\partial X}\right) d X+\left(\sum_{i=1}^{r} \beta_{i} Z \prod_{\substack{j=1 \\
j \neq i}}^{r} F_{j} \frac{\partial F_{i}}{\partial Y}\right) d Y \\
& +\left(\sum_{i=1}^{r} \beta_{i} Z \prod_{\substack{j=1 \\
j \neq i}}^{r} F_{j} \frac{\partial F_{i}}{\partial Z}-\left(\sum_{i=1}^{r} \beta_{i} \operatorname{deg}\left(F_{i}\right)\right) \prod_{j=1}^{r} F_{j}\right) d Z .
\end{aligned}
$$

Denote by $\mathbb{Q}^{+}$the set of positive rational numbers. It is straightforward to check that, for each $\bar{\beta} \in\left(\mathbb{Q}^{+}\right)^{r}$, the vector field $\mathcal{X}^{\prime}(\bar{\beta})$ has $\prod_{i=1}^{r} F_{i}^{n_{i}} / Z^{m}$ as a first integral, where $n_{i}=e \beta_{i}, 1 \leq i \leq r, e$ is the least common multiple of the denominators of the irreducible expressions of the rational numbers $\beta_{1}, \beta_{2}, \ldots, \beta_{r}$ and $m=\sum_{i=1}^{r} n_{i} \operatorname{deg}\left(F_{i}\right)$.

By $[12$, Lemma 1 (iv)], the pencil of curves defined by the equations

$$
\left\{\lambda_{1} \prod_{i=1}^{r} F_{i}^{n_{i}}+\lambda_{2} Z^{m}=0 \mid\left(\lambda_{1}: \lambda_{2}\right) \in \mathbb{C P}^{1}\right\}
$$

has exactly two elements which are not integral (reduced and irreducible) curves. These curves are those with equations $\prod_{i=1}^{r} F_{i}^{n_{i}}=0$ and $Z^{m}=0$. We must recall that this result holds when the curves $F_{i}=0$ have only one place at infinity. Otherwise this number may be larger [23].

Now, $\Omega(\bar{\beta})$ is reduced for all $\bar{\beta} \in\left(\mathbb{Q}^{+}\right)^{r}$ because, otherwise, the formula relating the degrees of the pencil and the form, and the factorization of the remarkable curves $[30$, Lemma 1.2], does not hold. 
Consider now the function $\omega(\bar{\beta})$ which maps any element $\bar{\beta} \in \mathbb{R}^{r}$ to the affine 1-form $\omega(\bar{\beta})=a_{\bar{\beta}}(x, y) d x+b_{\bar{\beta}}(x, y) d y$, where

$$
a_{\bar{\beta}}(x, y):=\sum_{i=1}^{r} \beta_{i} \prod_{\substack{j=1 \\ j \neq i}}^{r} f_{j} \frac{\partial f_{i}}{\partial x} \text { and } b_{\bar{\beta}}(x, y):=\sum_{i=1}^{r} \beta_{i} \prod_{\substack{j=1 \\ j \neq i}}^{r} f_{j} \frac{\partial f_{i}}{\partial y} .
$$

Regarding $a_{\bar{\beta}}(x, y)$ and $b_{\bar{\beta}}(x, y)$ as polynomials (with coefficients in a suitable ring) in the indeterminate $x$ (respectively, $y$ ), we can compute the resultant $\operatorname{Res}_{x}\left(a_{\bar{\beta}}, b_{\bar{\beta}}\right)$ (respectively, $\left.\operatorname{Res}_{y}\left(a_{\bar{\beta}}, b_{\bar{\beta}}\right)\right)$. The fact that $\Omega(\bar{\beta})$ is reduced for vectors $\bar{\beta} \in\left(\mathbb{Q}^{+}\right)^{r}$ implies the same fact for $\omega(\bar{\beta})$, which proves that $\operatorname{Res}_{x}\left(a_{\bar{\beta}}, b_{\bar{\beta}}\right) \in \mathbb{C}\left[\beta_{1}, \beta_{2}, \ldots, \beta_{r}\right][y]$ (respectively, $\operatorname{Res}_{y}\left(a_{\bar{\beta}}, b_{\bar{\beta}}\right) \in$ $\left.\mathbb{C}\left[\beta_{1}, \beta_{2}, \beta_{2}, \ldots, \beta_{r}\right][x]\right)$ is a nonzero polynomial. As a consequence, setting $\mathbb{R}^{+}$the set of positive real numbers, the 1 -form $\omega(\bar{\alpha})$ is reduced for all values $\bar{\alpha} \in\left(\mathbb{R}^{+}\right)^{r}$ such that $\operatorname{Res}_{x}\left(a_{\bar{\alpha}}, b_{\bar{\alpha}}\right) \neq 0$ and $\operatorname{Res}_{y}\left(a_{\bar{\alpha}}, b_{\bar{\alpha}}\right) \neq 0$. This proves that the 1 -form $\Omega_{\mathcal{X}}$ is reduced for generic exponents $\alpha_{1}, \alpha_{2}, \ldots, \alpha_{r} \in \mathbb{R}^{+}$and concludes the proof.

Now we state one of our main results, which determines the configuration $\mathcal{E}_{\infty}(\mathcal{X})$ corresponding to projective vector fields $\mathcal{X}$ having a DPWAI first integral with generic exponents.

Theorem 4.6. Assume that $\mathcal{X}$ is the complex projectivization of a polynomial vector field $\mathbf{X}$ having a DPWAI first integral as above whose exponents $\alpha_{1}, \alpha_{2}, \ldots, \alpha_{r}$ are generic. Then the following equality of configurations holds:

$$
\mathcal{E}_{\infty}(\mathcal{X})=\bigcup_{i=1}^{r}\left(\mathcal{B P}\left(\mathcal{P}_{i}\right) \cup\left\{S_{i}\right\} \cup \mathcal{J}_{i}\right),
$$

where, for $1 \leq i \leq r$, the $S_{i}$ 's are the points defined in Proposition 3.3 and each $\mathcal{J}_{i}=$ $\left\{R_{1 i}, R_{2 i}, \ldots\right\}$ is an infinite chain of infinitely near points such that $R_{1 i}$ (respectively, $R_{j i}$, $j \geq 2$ ) is a point that belongs to the exceptional divisor obtained by blowing-up $S_{i}$ (respectively, $\left.R_{j-1, i}\right)$. Moreover, the proximity graph of each chain $\left\{S_{i}\right\} \cup \mathcal{J}_{i}$ is that determined by the irrational number $\delta_{i} / \alpha_{i}$ and named $\operatorname{Prox}\left(\delta_{i} / \alpha_{i}\right)$, where

$$
\delta_{i}:=\sum_{\substack{j=1 \\ j \neq i}}^{r} \alpha_{j} \rho_{j i}
$$

and $\rho_{j i}$ are the integers $\sum_{Q}\left(C_{j}, C_{i}\right)_{Q}$ defined in (5).

Proof. Consider the vector $\bar{\alpha}=\left(\alpha_{1}, \alpha_{2}, \ldots, \alpha_{r}\right) \in\left(\mathbb{R}^{+}\right)^{r}$ given by some generic elements $\left\{\alpha_{i}\right\}_{i=1}^{r}$. Let $B:=B_{\bar{\alpha}}=\left\{\bar{\beta}_{n}=\left(\beta_{1 n}, \beta_{2 n}, \ldots, \beta_{r n}\right)\right\}_{n=1}^{\infty}$ be a sequence of vectors in $\left(\mathbb{Q}^{+}\right)^{r}$ such that $\lim _{n \rightarrow \infty} \bar{\beta}_{n}=\bar{\alpha}$ and, for each positive integer $n$, denote by $\mathcal{X}_{n}^{B}$ the vector field on $\mathbb{C P}^{2}$ given by the homogeneous 1-form $\Omega\left(\bar{\beta}_{n}\right)$ defined as in the proof of Proposition 4.5. Notice that each vector field $\mathcal{X}_{n}^{B}$ has the following rational first integral:

$$
\frac{\prod_{i=1}^{n} F_{i}^{e_{n} \beta_{i n}}}{Z^{m_{\bar{\beta}_{n}}}}
$$

where $e_{n}$ is the least common multiple of the denominators of the irreducible expressions of $\beta_{1 n}, \beta_{2 n}, \ldots, \beta_{r n}$ and

$$
m_{\bar{\beta}_{n}}:=\sum_{i=1}^{r} e_{n} \beta_{i n} \operatorname{deg}\left(F_{i}\right) .
$$


Hence, the restriction of $\mathcal{X}_{n}^{B}$ to the affine chart given by the complement of the line $Z=0$ has a WAI first integral. Notice that the dicritical configurations of the vector fields $\mathcal{X}_{n}^{B}$, $n \geq 1$, contain the configuration of infinitely near points of $\mathbb{C P}^{2}: \bigcup_{i=1}^{r}\left(\mathcal{B P}\left(\mathcal{P}_{i}\right) \cup\left\{S_{i}\right\}\right)$ (see Remark 3.4), and also that

$$
\mathcal{D}\left(\mathcal{X}_{n}^{B}\right) \cap \mathbb{C P}^{2}=\mathcal{E}_{\infty}(\mathcal{X}) \cap \mathbb{C P}^{2}
$$

by the proof of Proposition 4.5.

Let $\mathcal{P}_{n}^{B}$ be the pencil of curves defined by the equations

$$
\lambda_{1} \prod_{i=1}^{n} F_{i}^{e_{n} \beta_{i n}}+\lambda_{2} Z^{m_{\bar{\beta}_{n}}}=0
$$

where $\left(\lambda_{1}: \lambda_{2}\right) \in \mathbb{C P}^{1}$. By Proposition 3.3, the configuration of base points of this pencil, which coincides with $\mathcal{D}\left(\mathcal{X}_{n}^{B}\right)[24$, Corollary 1$]$, is given by

$$
\bigcup_{i=1}^{r}\left(\mathcal{B P}\left(\mathcal{P}_{i}\right) \cup\left\{S_{i}\right\} \cup \mathcal{L}_{i n}^{B}\right)
$$

where $\left\{S_{i}\right\} \cup \mathcal{L}_{\text {in }}^{B}$ is the configuration of base points of the (local) pencil (at $S_{i}$ ) defined by the non-zero linear combinations of $u^{e_{n} \beta_{i n}}$ and $t^{\delta_{i n}^{B}}, u, t$ being coordinates whose vanishing give local equations of the strict transform of $C_{i}$ at $S_{i}$ and of the exceptional divisor containing $S_{i}$, and where

$$
\delta_{i n}^{B}:=e_{n} \sum_{\substack{j=1 \\ j \neq i}}^{r} \beta_{j n} \rho_{j i} .
$$

Notice that, since $(u, t)$ is a regular system of parameters of the local ring at $S_{i}[12$, Lemma 1], the proximity graph of the configuration $\left\{S_{i}\right\} \cup \mathcal{L}_{i n}^{B}$ is

$$
\operatorname{Prox}\left(\frac{\delta_{i n}^{B}}{e_{n} \beta_{i n}}\right) \text {. }
$$

Now consider the configuration of infinitely near points of $\mathbb{C P}^{2}$

$$
\mathcal{B}:=\bigcup_{i=1}^{r}\left(\mathcal{B P}\left(\mathcal{P}_{i}\right) \cup\left\{S_{i}\right\}\right) .
$$

The reduction of singularities of $\mathcal{X}$ shows that $\mathcal{B} \subseteq \mathcal{E}_{\infty}(\mathcal{X})$ because $\bar{\alpha}$ is taken to be generic. In addition, $S_{1}, S_{2}, \ldots, S_{r}$ are the unique common points of $\mathcal{E}_{\infty}(\mathcal{X})$ and the surface $V$ obtained by blowing-up the points in $\cup_{i=1}^{r} \mathcal{B} \mathcal{P}\left(\mathcal{P}_{i}\right)$. This statement is a consequence of the forthcoming Lemma 4.7, [24, Lemma 1] (where it is proved that, under our conditions, blowing-up and taking associated 1-forms are commuting operations) and the fact that the local equation of the strict transform of $\mathcal{X}$ at any point of $V$ is the limit (when $n$ tends to infinity) of the local equations of the strict transforms of $\mathcal{X}_{n}^{B}$.

To finish our proof, we give a complete description of $\mathcal{E}_{\infty}(\mathcal{X})$. Notice that by $[24$, Lemma 1] the 1-form

$$
e_{n} \beta_{i n} t d u-\delta_{i n}^{B} u d t, \quad 1 \leq i \leq r,
$$

locally defines the strict transform of $\mathcal{X}_{n}^{B}$ at $S_{i}$, where $u=0$ is a local equation of the strict transform of $C_{i}$ at $S_{i}$ and $t=0$ is a local equation of the exceptional divisor. Then, taking limits, the 1-form

$$
t d u-\frac{\delta_{i}}{\alpha_{i}} d t
$$


defines the strict transform of $\mathcal{X}$ at $S_{i}$. From this local expression of the vector field, it is straightforward to deduce that the configuration of infinitely near points to $S_{i}$ belonging to $\mathcal{E}_{\infty}(\mathcal{X})$ is an infinite chain whose proximity graph is $\operatorname{Prox}\left(\delta_{i} / \alpha_{i}\right)$. In fact, this chain is infinite because Condition (3) in Definition 4.1 implies the irrationality of $\delta_{i} / \alpha_{i}$ for all $i \in\{1,2, \ldots, r\}$.

We conclude this section by stating and proving the above used Lemma 4.7, which allows us to prove that, with the notation as in the proof of the above theorem, the surface $V$ obtained by blowing-up the points in $\cup_{i=1}^{r} \mathcal{B P}\left(\mathcal{P}_{i}\right)$ has no points in $\mathcal{E}_{\infty}(\mathcal{X})$ different from the $S_{i}$ 's, $1 \leq i \leq r$.

The proof of Lemma 4.7 will require to use some objects of algebraic geometry which we briefly summarize for convenience of the reader. We will consider divisors on a surface $W$ and the Nerón-Severi group of $W, \mathrm{NS}(W)$, which is the group of numerical (equivalently, linear, in our case) equivalence classes $[C]$ of divisors $C$ on $W$. Recall that two divisors in $W$ are linearly equivalent whenever its difference is principal [31]. One can transform this group into an $\mathbb{R}$-linear space by using tensor product, $\mathrm{NS}(W) \otimes \mathbb{R}$, and then the cone of curves of $W$ is defined as

$\mathrm{NE}(W)=\left\{\sum a_{i}\left[C_{i}\right] \mid C_{i}\right.$ is a reduced and irreducible curve on $W, a_{i} \in \mathbb{R}$ and $\left.a_{i} \geq 0\right\}$.

The topological closure of $\mathrm{NE}(W)$ in $\mathrm{NS}(W) \otimes \mathbb{R}, \overline{\mathrm{NE}(W)}$, is the so-called closed cone of curves of $W$. Extremal rays of cones $\overline{\mathrm{NE}(X)}$ of algebraic varieties $X$ are crucial objects in the model minimal program in algebraic geometry $[38,33,7]$.

Lemma 4.7. Keep the notation as in Theorem 4.6 and its proof. Let $\pi: W \rightarrow \mathbb{C P}^{2}$ be the composition of the blowing-ups centered at the points of the dicritical configuration, $\mathcal{D}\left(\mathcal{X}_{n}^{B}\right)$, of the vector field on $\mathbb{C P}^{2}$ defined by $\Omega\left(\bar{\beta}_{n}\right)$. Consider the set $\left\{Q_{1}, Q_{2}, \ldots, Q_{r}\right\}$ of maximal points of $\mathcal{D}\left(\mathcal{X}_{n}^{B}\right)$ and the union $\Gamma=\bigcup_{i=1}^{r} \tilde{E}_{Q_{i}}$ of the strict transforms on $W$ of the exceptional divisors obtained by blowing-up the points $Q_{i}, 1 \leq i \leq r$.

Then, the singularities of the strict transform of $\mathcal{X}_{n}^{B}$ on $W$ which belong to the exceptional locus of $\pi$, but not to $\Gamma$, are simple and the local equation of $\mathcal{X}_{n}^{B}$ at any of them has the form

$$
a t_{2} d t_{1}+b t_{1} d t_{2}
$$

where $a, b \in \mathbb{Q}^{+} \cup\{0\}, a+b \neq 0$ and, $t_{1}=0$ (respectively, $t_{2}=0$ ) is the local equation, at the singularity, of the strict transform of an irreducible exceptional divisor (respectively, the line at infinity $Z=0$ ).

Proof. First of all notice that, by Proposition 3.3, the dicritical configuration of $\mathcal{X}_{n}^{B}$ and the base points configuration of $\mathcal{P}_{n}^{B}$ coincide; i.e., $\mathcal{D}\left(\mathcal{X}_{n}^{B}\right)=\mathcal{B} \mathcal{P}\left(\mathcal{P}_{n}^{B}\right)$.

Let $H$ be an arbitrary irreducible component of a curve of the pencil $\mathcal{P}_{n}^{B}$, different from $Z=0$, and denote by $\tilde{H}$ its strict transform on $W$. To prove the lemma, it is enough to show that, outside $\Gamma, \tilde{H}$ has no singularity on the exceptional part of $\pi$.

We can assume $H \neq C_{i}$ for all $i \in\{1,2, \ldots, r\}$ because $\pi$ is a common resolution of the singularities at infinity of these curves (see [12, Lemma 1 (iii)] and $[2,37]$ ).

Notice that the self-intersection of $\tilde{H}$ cannot be negative because, in this case, the class of $\tilde{H}$ in $N S(W) \otimes \mathbb{R}$ would generate an extremal ray of the closed cone of curves of $W$ [34, Lemma 1.22], and this is not possible by [12, Theorem 3]. Hence, $\tilde{H}^{2} \geq 0$.

The pencil $\mathcal{P}_{n}^{B}$ defines a rational map $\mathbb{C P}^{2} \cdots \rightarrow \mathbb{C P}^{1}$, that is a morphism from an open set of $\mathbb{C P}^{2}$ to $\mathbb{C P}^{1}$ which cannot be extended to any larger open set. The elimination of its indeterminacies induces a morphism $f: W \rightarrow \mathbb{C P}^{1}$ contracting to a point the strict 
transform of any curve in the pencil [6, Theorem II.7]. In particular, it contracts $\tilde{H}$. The morphism $f$ is defined by the global sections of the invertible sheaf [31] associated to the divisor $D$ on $W$ given by the strict transform of a general curve of the pencil $\mathcal{P}_{n}^{B}$. Notice that $D^{2}=0$ because the strict transforms on $W$ of two different general curves of the pencil do not meet. Then,

$$
D \cdot \tilde{H}=0
$$

(and $\tilde{H}^{2} \geq 0$ by the previous paragraph). Hence $\tilde{H}$ must be linearly equivalent to $D[6$, Lemma III.9].

To conclude the proof, we take into account that the exceptional irreducible divisors which are not contracted by the morphism $f$ are the irreducible components of $\Gamma[12$, Lemma 1 (iii)], and this implies that, if $E$ is the strict transform of an irreducible exceptional divisor, then

$$
\tilde{H} \cdot E=D \cdot E>0
$$

if and only if $E$ is contained in $\Gamma$. This proves that $\tilde{H}$ can only have singularities on $\Gamma$ and the lemma.

\section{THE ALGORITHM}

In this last section we state the previously mentioned algorithm which computes a first integral of planar polynomial vector fields having a DPWAI first integral with generic exponents.

In [18], Darboux proved the existence of a first integral for polynomial vector fields with enough invariant algebraic curves. In this case, he also gave a procedure to compute it. This result was improved in [17] (see also [16]). Next we state the Darboux theorem.

Theorem 5.1. Let $\mathbf{X}$ be the differential system in (2). Suppose that the bivariate complex polynomials $f_{i}:=f_{i}(x, y), 1 \leq i \leq r$, define algebraic curves which are irreducible and invariant for $\mathbf{X}$. Then

(i) $H=f_{1}^{\lambda_{1}} \cdots f_{r}^{\lambda_{r}}$, where $\lambda_{i} \in \mathbb{C}, 1 \leq i \leq r$, are not all zero, is a first integral of $\mathbf{X}$ if, and only if, the linear combination

$$
\sum_{i=1}^{r} \lambda_{i} k_{i}(x, y)
$$

vanishes, $k_{i}(x, y)$ being the cofactor of the invariant curve defined by $f_{i}(x, y), 1 \leq$ $i \leq r$.

(ii) There is an identically zero linear combination $\sum_{i=1}^{r} \lambda_{i} k_{i}(x, y)$ with not all zero coefficients $\lambda_{i}$ whenever $r=\left(\begin{array}{c}d+1 \\ 2\end{array}\right)+1$.

As a consequence of the above result, a key fact for obtaining first integrals is the searching of invariant algebraic curves. Notice that it is a very hard problem.

Next we present Theorem 5.2 which, together with some previous results, allows us to state our algorithm. This algorithm provides enough invariant curves to apply Theorem 5.1 and determine a Darboux first integral in case the input we supply is a polynomial vector field having a DPWAI first integral with generic exponents.

The mentioned theorem will use a cluster $\left(\mathcal{K}, \mathbf{m}_{\mathcal{K}}\right)$ attached to any finite chain $\mathcal{K}$ of infinitely near points of $\mathbb{C P}^{2}$. Recall that a configuration $\mathcal{K}=\left\{Q_{1}, Q_{2}, \ldots, Q_{s}\right\}$ is a chain if $Q_{i}, i>1$, belongs to the exceptional divisor created by $Q_{i-1}$. The sequence of positive 
integers $\mathbf{m}_{\mathcal{K}}:=\left(m_{Q}\right)_{Q \in \mathcal{K}}$ is defined as follows: $m_{Q}=1$ if $Q$ is the maximal point of $\mathcal{K}$ and $m_{Q}=\sum m_{R}$ otherwise, where the sum runs through the set of points $R$ in $\mathcal{K}$ which are proximate to $Q$. Also, given an arbitrary configuration $\mathcal{C}$ and any point $Q \in \mathcal{C}$, we will denote by $\mathcal{C}_{Q}$ the finite chain defined by those points $R$ in $\mathcal{C}$ such that $Q$ is infinitely near to $R$.

Theorem 5.2. Let $\mathcal{X}$ be a projective vector field and $\alpha_{1}, \alpha_{2}, \ldots, \alpha_{r}$ real numbers as in Theorem 4.6. Keep the above notation and consider the clusters $\mathcal{K}_{i}:=\left(\mathcal{C}_{i}, \mathbf{m}_{\mathcal{C}_{i}}\right), 1 \leq i \leq r$, where $\mathcal{C}_{i}=\left\{L_{i 0}, L_{i 1}, \ldots, L_{i \ell_{i}}:=S_{i}\right\}:=\mathcal{C}_{S_{i}}=\mathcal{B P}\left(\mathcal{P}_{i}\right) \cup\left\{S_{i}\right\}$. Set $\mathbf{m}_{\mathcal{C}_{i}}=\left(m_{Q}\right)_{Q \in \mathcal{C}_{i}}$. Then the following equalities hold:

(i) $\left[\operatorname{deg}\left(C_{i}\right)\right]^{2}-\sum_{j=0}^{\ell_{i}} m_{L_{i j}}^{2}=-1$, and

(ii) $\operatorname{deg}\left(C_{i}\right)=\sum_{j=0}^{\kappa_{i}} m_{L_{i j}}$,

where $\kappa_{i}$ denotes the largest index $j$ such that the strict transform of the line at infinity passes through $L_{i j}$. Moreover, $C_{i}$ is the unique curve in the linear system $\mathcal{L}_{\sum_{j=1}^{\kappa_{i}} m_{L_{i j}}}\left(\mathcal{K}_{i}\right)$.

Proof. Let $U$ be the surface we get by blowing-up $\mathcal{B P}\left(\mathcal{P}_{i}\right)$. Consider the line at infinity $L$ and two different general curves $\Delta_{1}$ and $\Delta_{2}$ of the pencil $\mathcal{P}_{i}$. Then, their strict transforms on $U, \tilde{L}, \tilde{\Delta}_{1}$ and $\tilde{\Delta}_{2}$, do not meet. Therefore $\tilde{\Delta}_{1} \cdot \tilde{\Delta}_{2}=0$ and $\tilde{\Delta}_{1} \cdot \tilde{L}=0$. Both equalities prove, respectively, the equalities (i) and (ii) after noticing that $\tilde{\Delta}_{1}$ and $\tilde{\Delta}_{2}$ are linearly equivalent to the strict transform of $C_{i}$.

We conclude the proof by noticing that our last assertion follows from (i) and Bézout Theorem [6, I.9 (a)].

Now we present our algorithm which, applied to a polynomial vector field, computes candidates to be the polynomials $f_{1}, f_{2}, \ldots, f_{r}$ appearing in a DPWAI first integral. Theorems 4.6 and 5.2 prove that when the input has a DPWAI first integral with generic exponents, the output will be the mentioned polynomials $f_{i}$.

We will need the following notation: given an arbitrary configuration $\mathcal{C}$ we define, for each maximal point $Q$ of $\mathcal{C}$, the integer $I_{Q}(\mathcal{C}):=d_{Q}(\mathcal{C})^{2}-\sum_{P \in \mathcal{C}_{Q}} m_{P}^{2}$, where $\mathbf{m}_{\mathcal{C}_{Q}}=$ $\left(m_{P}\right)_{P \in \mathcal{C}_{Q}}$ and $d_{Q}(\mathcal{C}):=\sum m_{P}$, the sum being taken over the points $P$ in $\mathcal{C}_{Q}$ such that the strict transform of the line at infinity passes through $P$.

\section{Algorithm 5.3.}

- Input: An arbitrary polynomial vector field $\mathbf{X}$.

- Output: Either a finite set $\left\{f_{i}(x, y)\right\}_{i=1}^{r}$ of polynomials in two indeterminates which are candidates for applying Theorem 5.1 and obtaining a Darboux first integral, or 0 .

(1) Compute an homogeneous 1-form defining the complex projectivization $\mathcal{X}$ of $\mathbf{X}$.

(2) Compute the set $\Omega^{\prime}$ consisting of the points $Q$ in the singular configuration $\mathcal{S}(\mathcal{X})$ which are infinitely near to a point of the line at infinity.

(3) Let $Q_{1}, Q_{2}, \ldots, Q_{\ell}$ be the maximal points of $\Omega^{\prime}$. For every $i \in\{1,2, \ldots, \ell\}$ compute the maximal configuration $\Omega^{i}$ of points $P$ infinitely near to $Q_{i}$ satisfying the following conditions:

(a) $P$ is free,

(b) $P \in \mathcal{E}_{\infty}(\mathcal{X})$ (that is, $P$ is a simple singularity of the strict transform of the vector field $\mathcal{X}$ whose associated quotient of eigenvalues is a positive irrational number),

(c) $I_{P}\left(\mathcal{E}_{\infty}(\mathcal{X})\right) \geq-1$ 
If $\Omega^{i}$ is empty for some $i \in\{1,2, \ldots, \ell\}$, then return 0 . Else, define $\Omega:=\Omega^{\prime} \cup \Omega^{1} \cup$ $\cdots \cup \Omega^{\ell}$ and go to Step (4).

(4) Let $M=\left\{S_{1}, S_{2}, \ldots, S_{r}\right\}$ be the set of maximal points of $\Omega$. If $I_{S_{i}}\left(\mathcal{E}_{\infty}(\mathcal{X})\right) \neq-1$ for some $i \in\{1,2, \ldots, r\}$ then return 0 . Else go to Step (5).

(5) If the linear systems $\mathcal{L}_{d_{S_{i}}}\left(\Omega_{S_{i}}, \mathbf{m}_{\Omega_{S_{i}}}\right)$ have projective dimension 0 for all $i \in$ $\{1,2, \ldots, r\}$ (where $d_{S_{i}}:=d_{S_{i}}\left(\mathcal{E}_{\infty}(\mathcal{X})\right)$ ), then return

$$
\left\{F_{1}(x, y, 1), F_{2}(x, y, 1), \ldots, F_{r}(x, y, 1)\right\},
$$

$F_{i}(X, Y, Z)$ being an homogeneous polynomial defining the unique curve in

$$
\mathcal{L}_{d_{S_{i}}}\left(\Omega_{S_{i}}, \mathbf{m}_{\Omega_{S_{i}}}\right)
$$

Else, return 0 .

Our procedure to decide about DPWAI integrability of a vector field $\mathbf{X}$ has two steps. First we run Algorithm 5.3 with input $\mathbf{X}$ and, when the output is not 0 , we get $r$ curves defined by equations $f_{i}=0,1 \leq i \leq r$. These curves aspire to be invariant of $\mathbf{X}$. If yes, we compute their cofactors

$$
k_{i}=\frac{p \frac{\partial f_{i}}{\partial x}+q \frac{\partial f_{i}}{\partial y}}{f_{i}},
$$

and then, we test the existence of values $\lambda_{i} \in \mathbb{R}^{+}, 1 \leq i \leq r$, for which the polynomial in (7) vanishes. In the positive case, the function $H$ in Theorem 5.1 is a first integral of $\mathbf{X}$. Notice that the above checking only involves the resolution of a homogeneous linear system with $r$ variables and $\left(\begin{array}{c}d+1 \\ 2\end{array}\right)$ equations.

When the input $\mathbf{X}$ has a DPWAI first integral with generic exponents, we also obtain its extended resolution of singularities over the line at infinity. Otherwise, $\mathbf{X}$ has not a DPWAI first integral with generic exponents, the output of Algorithm 5.3 could be 0 or some non necessarily invariant curves by $\mathbf{X}$. However, the output of Algorithm 5.3 could also provide enough invariant curves and then, we would obtain a DPWAI first integral by means of Theorem 5.1 .

We conclude this paper with an example where we detail our procedure for computing a Darboux first integral of the mentioned class of polynomial vector fields.

Example 5.4. Consider the polynomial vector field

$$
\mathbf{X}=a(x, y) d x+b(x, y) d y,
$$

where

$a(x, y)=(3+4 \pi) x^{6} y^{2}+(3+\sqrt{2}+4 \pi) x^{7}+4 \pi x^{3} y^{3}+(\sqrt{2}+4 \pi) x^{4} y-3 x^{2} y^{3}-(3+\sqrt{2}) x^{3} y-\sqrt{2} y^{2}$ and

$b(x, y)=2 \sqrt{2} x^{7} y+(1+2 \sqrt{2}) x^{4} y^{2}+x^{5}-(2 \sqrt{2}+\pi) x^{3} y^{2}-\pi x^{4}-(1+2 \sqrt{2}+\pi) y^{3}-(1+\pi) x y$.

Algorithm 5.3 gives rise to a configuration of infinitely near points of $\mathbb{C P}^{2}, \Omega=\left\{P_{i}\right\}_{i=1}^{18}$, which has 3 maximal points:

$$
S_{1}=P_{9}, S_{2}=P_{13} \text { and } S_{3}=P_{18} .
$$

The proximity graph of the configuration is displayed in Figure 2. Moreover, the multiplicity sequences are

$$
\begin{gathered}
\mathbf{m}_{\Omega_{S_{1}}}=(3,1,1,1,1,1,1,1,1), \\
\mathbf{m}_{\Omega_{S_{2}}}=(2,1,1,1,1,1,1),
\end{gathered}
$$




$$
\mathbf{m}_{\Omega_{S_{3}}}=(1,1,1,1,1)
$$

and, since the strict transforms of the line at infinity pass through $P_{1}$ and $P_{2}, d_{S_{1}}=4$, $d_{S_{2}}=3, d_{S_{3}}=2$ and $I_{S_{i}}=-1$ for all $i \in\{1,2,3\}$. In addition the algorithm allows us to determine that

$$
\mathcal{L}_{d_{i}}\left(\Omega_{S_{i}}, \mathbf{m}_{\Omega_{S_{i}}}\right)=\left\{C_{i}\right\}
$$

where $C_{1}$ (respectively, $C_{2}, C_{3}$ ) is the projective curve (having only one place at infinity) with equation $X^{4}-Y Z^{3}=0$ (respectively, $X^{3}+Y Z^{2}=0, Y^{2}+X Z=0$ ). In fact, Algorithm 5.3 returns the set $\left\{f_{1}, f_{2}, f_{3}\right\}$, where $f_{1}(x, y)=x^{4}-y, f_{2}(x, y)=x^{3}+y$ and $f_{3}(x, y)=y^{2}+x$.

Now, applying Theorem 5.1, one obtains that $f_{1}^{\pi} f_{2} f_{3}^{\sqrt{2}}$ is a DPWAI first integral of $\mathbf{X}$.

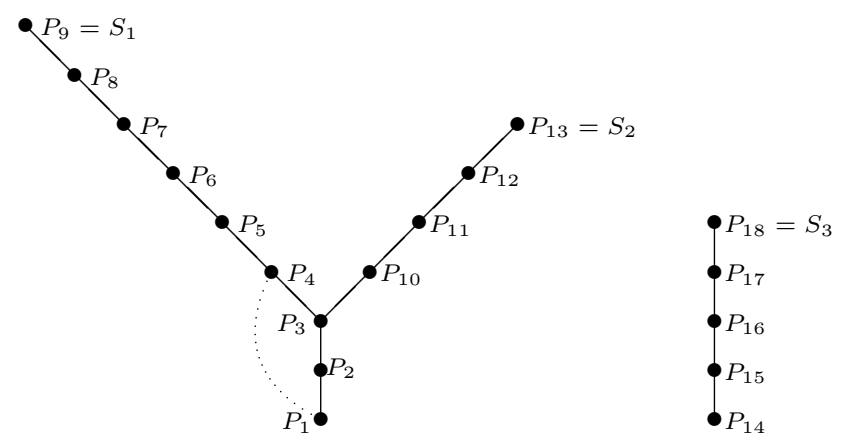

FIgURE 2. Proximity graph of the configuration obtained applying Algorithm 5.3.

Finally, we notice that, with notations as in Theorem 4.6,

$$
\delta_{1}=4+8 \sqrt{2}, \delta_{2}=6 \sqrt{2}+4 \pi \text { and } \delta_{3}=6+8 \pi .
$$

Therefore, the proximity graph of the extended reduction of singularities over the line at infinity, $\mathcal{E}_{\infty}(\mathcal{X})$, is obtained from that in Figure 2 by adding three infinite chains $\mathcal{J}_{1}$, $\mathcal{J}_{2}$ and $\mathcal{J}_{3}$ over $S_{1}, S_{2}$ and $S_{3}$ such that the proximity graph of $\mathcal{J}_{1} \cup\left\{S_{1}\right\}$ (respectively, $\left.\mathcal{J}_{2} \cup\left\{S_{2}\right\}, \mathcal{J}_{3} \cup\left\{S_{3}\right\}\right)$ is $\operatorname{Prox}\left(\frac{4+8 \sqrt{2}}{\pi}\right)$ (respectively, $\operatorname{Prox}(6 \sqrt{2}+4 \pi)$, $\left.\operatorname{Prox}\left(\frac{6+8 \pi}{\sqrt{2}}\right)\right)$. To complete our example we show, in Figure 3, the bottom part of the proximity graph of the chain $\mathcal{J}_{1} \cup\left\{S_{1}\right\}$, without labels. A similar procedure provides the remaining chains.

\section{REFERENCES}

[1] S. S. Abhyankar, Lectures on expansion techniques in Algebraic Geometry, Tata Institute of Fundamental Research Lectures on Mathematics and Physics 57, Tata Institute of Fundamental Research, Bombay (1977).

[2] S. S. Abhyankar and T. T. Moh, Newton-Puiseux expansion and generalized Tschirnhausen transformation, J. Reine Angew. Math. 260 (1973), 47-83 and 261 (1973), 29-54.

[3] S. S. Abhyankar and T. T. Moh, "On the semigroup of a meromorphic curve", Proc. Int. Symp. Algebraic Geometry Kyoto (1977), 249-414.

[4] M.J. Álvarez, A. Ferragut and X. Jarque, A survey on the blow-up technique, Int. J. Bifur. Chaos 21 (2011), 3103-3118.

[5] L. Autonne, Sur la théorie des équations différentielles du premier ordre et du premier degré, J. Éc. Polytech. 61 (1891), 35-122; 62 (1892), 47-180.

[6] A. Beauville, Complex algebraic surfaces, London Math. Soc. Student Texts 34, Cambridge University Press, 1996. 


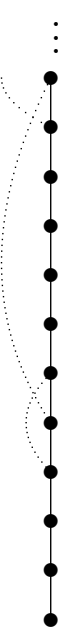

Figure 3. Proximity graph of the chain $\mathcal{J}_{1} \cup\left\{S_{1}\right\}$

[7] C. Birkar, et al. Existence of minimal models for varieties of log general type, J. Amer. Math. Soc. 23 (2010), 405-468.

[8] A. Bostan et al., Efficient algorithms for computing rational first integrals and Darboux polynomials of planar polynomial vector fields, Math. Comp. 85 (2016), 1393-1425.

[9] M. Brunella, Birational Geometry of Foliations, Springer, 2000.

[10] A. Campillo, G. González-Sprinberg and M. Lejeune-Jalabert. Clusters of infinitely near points, Math. Ann. 306 (1996), 169-194.

[11] A. Campillo, O. Piltant and A.J. Reguera, Cones of curves and of line-bundles on surfaces associated with curves having one place at infinity, Proc. London Math. Soc. 84 (2002), 559-580.

[12] A. Campillo, O. Piltant and A.J. Reguera, Cones of curves and of line bundles "at infinity" J. Algebra 293 (2005), 513-542.

[13] A. Campillo and J. Olivares, Polarity with respect to a foliation and Cayley-Bacharach Theorems, J. Reine Angew. Math. 534 (2001), 95-118.

[14] E. Casas-Alvero, Singularities of plane curves, London Math. Soc. Lect. Notes Series 276 (2000), Cambridge University Press.

[15] D. Cerveau and A. Lins-Neto, Holomorphic foliations in $\mathbb{C P}(2)$ having an invariant algebraic curve, Ann. Inst. Fourier 41 (4) (1991), 883-903.

[16] G. Chèze, Darboux theory of integrability in the sparse case, J. Diff. Eq. 257 (2014), 601-609.

[17] C. Christopher and J. Llibre, Integrability via invariant algebraic curves for planar polynomial differential systems, Ann. Diff. Eq. 16 (2000), 5-19.

[18] G. Darboux, Mémoire sur les équations différentielles algébriques du premier ordre et du premier degré (Mélanges), Bull. Sci. Math. 32 (1878), 60-96; 123-144; 151-200.

[19] F. Dumortier, Singularities of vector fields on the plane, J. Diff. Eq. 23 (1977), 53-106.

[20] F. Dumortier, J. Llibre and J.C. Artés. Qualitative theory of planar differential systems, UniversiText, Springer-Verlag, New York, 2006.

[21] C. Favre and M. Jonsson, Eigenvaluations, Ann. Sci. Éc. Norm. Sup. 40 (2007), 309-349.

[22] C. Favre and M. Jonsson, Dynamical compactifications of $\mathbb{C}^{2}$, Ann. Math. 173 (2011), 211-248.

[23] A. Ferragut, Some new results on Darboux integrable differential systems, J. Math. Anal. Appl. 394 (2012), 416-424.

[24] A. Ferragut, C. Galindo and F. Monserrat, A class of polynomial planar vector fields with polynomial first integral, J. Math. Anal. Appl. 430 (2015), 354-380.

[25] A. Ferragut and H. Giacomini, A new algorithm for finding rational first integrals of polynomial vector fields, Qual. Th. Dyn. Syst. 9 (2010), 89-99.

[26] C. Galindo and F. Monserrat, The Abhyankar-Moh theorem for plane valuations at infinity, J. Algebra 374 (2013), 3181-194. 
[27] C. Galindo and F. Monserrat, The Poincaré problem, algebraic integrability and dicritical divisors, J. Diff. Eq. 256 (2014), 3614-3633.

[28] C. Galindo and F. Monserrat, The cone of curves and the Cox ring of rational surfaces given by divisorial valuations, Adv. Math. 290 (2016), 1040-1061.

[29] C. Galindo, F. Monserrat and J.J. Moyano-Fernández, Minimal plane valuations, J. Alg. Geom. 27 (2018), 751-783.

[30] A. García Zamora, Foliations in algebraic surfaces having a rational first integral, Publ. Mat. 41 (1997), 357-373.

[31] R. Hartshorne, Algebraic Geometry, Springer-Verlag, 1977.

[32] J.P. Jouanolou, Equations de Pfaff algébriques, Lect. Notes Math. 708, Springer-Verlag, 1979.

[33] Y. Kawamata, The cone of curves of algebraic varieties, Ann. Math. 119 (1984), 603-633.

[34] J. Kollár and S. Mori, Birational geometry of rational varieties, Cambridge Tracts in Math. 134, Cambridge University Press, 1998.

[35] S. Lang, Algebra, Graduate Texts in Math. 211, Springer-Verlag, 2002.

[36] J. Llibre and C. Pantazi, Polynomial differential systems having a given Darbouxian first integral, Bull. Sci. Math. 128 (2004), 775-788.

[37] T.T. Moh, On analytic irreducibility at infinity of a pencil of curves, Proc. Amer. Math. Soc. 44 (1974), 22-23.

[38] S. Mori, Threefolds whose canonical bundles are not numerically effective, Ann. Math. 116 (1982), 133-176.

[39] P. Mondal, How to determine the sign of valuation on $\mathbb{C}[x, y]$, Michigan Math. J. 66 (2017), 227-244.

[40] P. Painlevé, "Sur les intégrales algébriques des équations différentielles du premier ordre" and "Mémoire sur les équations différentielles du premier ordre" in Ouvres de Paul Painlevé, Tome II, Éditions du Centre National de la Recherche Scientifique 15, quai Anatole-France, Paris 1974.

[41] J.V. Pereira, Vector fields, invariant varieties and linear systems, Ann. Inst. Fourier 51(5) (2001), $1385-1405$.

[42] H. Poincaré, Mémoire sur les courbes définies par les équations différentielles, J. Math. Pures Appl. 3 (7) (1881), 375-442; 3 (8) (1882), 251-296; 4 (1) (1885), 167-244; in Oeuvres de Henri Poincaré, vol. I, Gauthier-Villars, Paris 1951, 3-84, 95-114.

[43] H. Poincaré, Sur l'intégration algébrique des équations différentielles du premier ordre et du premier degré (I), Rend. Circ. Mat. Palermo 5 (1891), 161-191; (II) 11 (1897), 193-239.

[44] A. Seidenberg, Reduction of singularities of the differentiable equation $A d y=B d x$, Amer. J. Math. 90 (1968), 248-269.

[45] J. Xie, Intersections of valuation rings in k[x,y], Proc. London Math. Soc. 111 (2015), 240-274.

A. Ferragut: Universidad Internacional de la Rioja. Avenida de la Paz 137, 26006 Logroño, Spain

E-mail address: toni.ferragut@unir.net

C. Galindo: Instituto Universitario de Matemáticas y Aplicaciones de Castellón (imaC) and Departamento de Matemáticas, Universitat Jaume I, 12071 Castelló de la Plana, Spain E-mail address: galindo@uji.es

F. Monserrat: E.T.S. D’Informática Aplicada, Universitat Politècnica de València, 46002 VALÈnCIA, SPAIN

E-mail address: framonde@mat.upv.es 